\title{
Analysis of Nonlinear Stochastic Systems with Jumps Generated by Erlang Flow of Events
}

\author{
Alexander S. Kozhevnikov, Konstantin A. Rybakov ${ }^{*}$ \\ Department of Mathematical Cybernetics, Moscow Aviation Institute, Moscow, Russia \\ Email: *rkoffice@mail.ru
}

Received December 2, 2012; revised January 2, 2013; accepted January 9, 2013

\begin{abstract}
In this paper we consider the stochastic systems with jumps (random impulses) generated by Erlang flow of events that lead to discontinuities in paths. These systems may be used in various applications such as a control of complex technical systems, financial mathematics, mathematical biology and medicine. We propose to use a spectral method formalism to the probabilistic analysis problem for the stochastic systems with jumps. This method allows to get a solution of the analysis problem in an explicit form.
\end{abstract}

Keywords: Analysis; Erlang Flow of Events; Generalized Fokker-Planck Equations; Random Impulses; Jump-Diffusion Process; Spectral Characteristic; Spectral Method Formalism; Stochastic System

\section{Introduction}

In this paper we consider the stochastic systems with jumps generated by Erlang flow of events that lead to discontinuities of sample paths. These systems are called the jump-diffusion systems or the stochastic systems with random quantization period. Jumps may have different characteristics that describe intervals between them and their amplitudes [1,2].

Stochastic systems with jumps are used in various applications such as complex technical systems (control of moving objects, jam-resistant radars, radioisotope measuring systems, electrical circuits with impulse sources), financial mathematics (description of stock price movements and valuation of stock options), mathematical biology and medicine (biomass control and drug delivery model) [2,3].

The goal of this paper is to develop the spectral method [4-6] for a problem of the probabilistic analysis for jump-diffusion systems. The spectral method formalism has been used previously to the stochastic systems with jumps generated by Poisson flow of events. Here we consider more complex problem which assumes that we have Erlang flow of jumps. This allows to investigate the stochastic systems with jumps in sample paths at the random time moments. Intervals between these moments can be described by not only the exponential distribution, but Erlang distribution [7].

${ }^{*}$ Corresponding author.

\section{Problem Statement}

We assume that the system behavior is described by a jump-diffusion process. This process can be represented as a solution of the stochastic differential equation [1]:

$$
\begin{aligned}
& \mathrm{d} X(t)=f(t, X(t)) \mathrm{d} t+\sigma(t, X(t)) \mathrm{d} W(t)+\mathrm{d} Q(t), \\
& X\left(t_{0}\right)=X_{0},
\end{aligned}
$$

where $X \in R^{n}$ is a state vector, $t \in T, T=\left[t_{0}, t_{1}\right]$; $f(t, x): T \times R^{n} \rightarrow R^{n}, \sigma(t, x): T \times R^{n} \rightarrow R^{n \times s}, W(t)$ is an $s$-dimensional standard Wiener process independent of $X_{0}$.

The component $\mathrm{d} Q(t)$ describes "extreme events" attended by jumps in sample paths of the process $X(t)$ (e.g., a technical failure or a stock market crash). We assume that

$$
Q(t)=\sum_{i=1}^{J(t)} Y\left(\tau_{i}\right) .
$$

Here $J(t)$ is the $N$-th order Erlang process (Erlang process $J(t)$ is a "censored" Poisson process in which $N-1$ consecutive points are removed from a Poisson process $P(t)$ with the transition intensity $\lambda(t)$ and then one point left unchanged [7]). $Y\left(\tau_{\text {: }}\right)$ are independent random variables from $R^{n}$ whose distribution is given by the probability density function $\psi(t, y)$, i.e., state vector gets random increment at time moments $\tau_{1}, \tau_{2}, \cdots$ associated with Erlang flow of events [1]:

$$
X\left(\tau_{i}\right)=X\left(\tau_{i}-0\right)+Y\left(\tau_{i}\right)
$$


The process $Q(t)$ may be represented as

$$
Q(t)=\sum_{i=1}^{P(t)} \xi_{i} Y\left(\theta_{i}\right),
$$

where the value $\xi_{i}$ is used to "censor" $N-1$ Poisson flow events in succession and to select each event which is a multiple of $N\left(\xi_{i}\right.$ is the periodic function: $\left.\xi_{i+N}=\xi_{i}\right)$ :

$$
\xi_{i}=\left\{\begin{array}{l}
1, i(\bmod N)=0, \\
0, i(\bmod N) \neq 0 ;
\end{array}\right.
$$

time moments $\theta_{1}, \theta_{2}, \cdots$ conform to the events in Poisson flow: $\theta_{i N}=\tau_{i}$.

Introduce a stochastic process $K(t)$ with a finite state set $\{1,2, \cdots, N\}$. These states are replaced sequentially starting from 1 with the transition intensity $\lambda(t)$ :

$$
K(t)=1+P(t)(\bmod N) .
$$

when the state with number $N$ passes into the state with number 1 , the state vector $X$ gets a random increment which leads to a jump in sample paths of the process $X(t)$ (see Figure 1).

The introduction of the process $K(t)$ allows to represent the probability density function $\varphi(t, x)$ of the state vector $X$ as follows:

$$
\varphi(t, x)=\sum_{k=1}^{N} \varphi^{(k)}(t, x),
$$

where functions $\varphi^{(k)}(t, x)$ satisfy generalized Fokker-Planck equations $[2,5,8]$ :

$$
\begin{aligned}
\frac{\partial \varphi^{(1)}(t, x)}{\partial t} & =\mathcal{A} \varphi^{(1)}(t, x)-\lambda(t) \varphi^{(1)}(t, x) \\
& +\lambda(t) \int_{R^{n}} \psi(t, x-z) \varphi^{(N)}(t, z) \mathrm{d} z,
\end{aligned}
$$

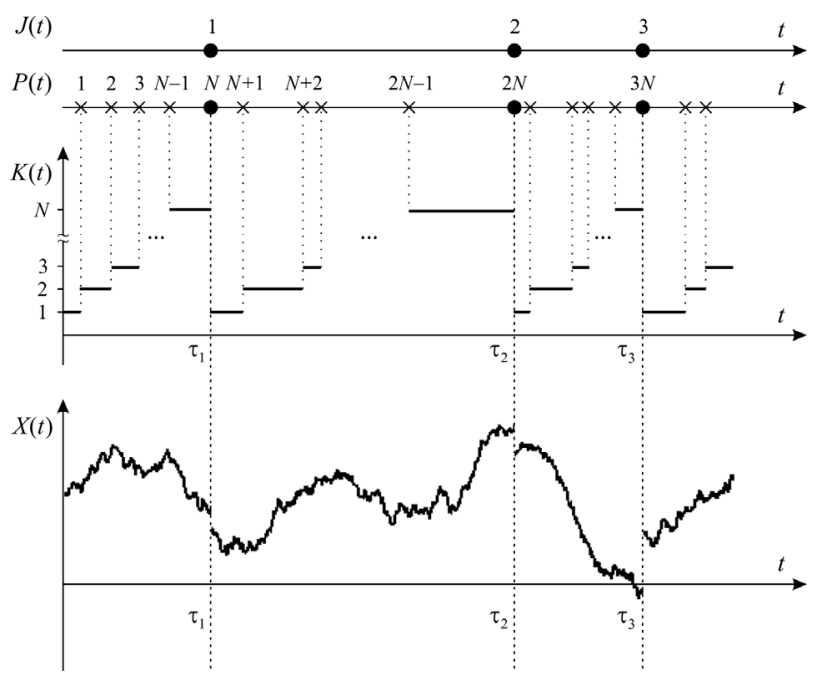

Figure 1. Sample paths of processes $K(t)$ and $X(t)$.

$$
\begin{aligned}
& \frac{\partial \varphi^{(k)}(t, x)}{\partial t} \\
& =\mathcal{A} \varphi^{(k)}(t, x)-\lambda(t) \varphi^{(k)}(t, x)+\lambda(t) \varphi^{(k-1)}(t, x), \\
& k=2, \cdots, N .
\end{aligned}
$$

Here

$$
\begin{aligned}
& \mathcal{A} \varphi^{(k)}(t, x) \\
= & -\sum_{i=1}^{n} \frac{\partial}{\partial x_{i}}\left[f_{i}(t, x) \varphi^{(k)}(t, x)\right] \\
& +\frac{1}{2} \sum_{i=1}^{n} \sum_{j=1}^{n} \frac{\partial^{2}}{\partial x_{i} \partial x_{j}}\left[g_{i j}(t, x) \varphi^{(k)}(t, x)\right], \\
k & =1,2, \cdots, N, \\
g_{i j}(t, x)= & \sum_{r=1}^{s} \sigma_{i r}(t, x) \sigma_{j r}(t, x), i, j=1,2, \cdots, n .
\end{aligned}
$$

The initial state $X_{0}$ is determined by a given probability density function $\varphi_{0}(x)$. The initial state of the process $K(t)$ is fixed: $K\left(t_{0}\right)=1$. So,

$$
\varphi^{(1)}\left(t_{0}, x\right)=\varphi_{0}(x), \varphi^{(k)}\left(t_{0}, x\right)=0, k=2, \cdots, N .
$$

The last term on the right side of Equation (2) can be written in the operator form:

$$
\mathcal{H} \varphi(t, x)=\lambda(t) \int_{R^{n}} \psi(t, x-z) \varphi(t, z) \mathrm{d} z
$$

for all admissible functions $\varphi(t, x) ; \mathcal{H}$ is a linear operator which is a composition of the multiplication operator and the Fredholm operator with kernel $\psi(t, x-z)$.

The analysis problem of the stochastic systems with jumps described by Equation (1) is to find the probability density function $\varphi(t, x)$ of the state vector $X$.

We assume that the unique solutions of Equation (1) and Equations (2)-(5) exist for given functions $f(t, x)$, $\sigma(t, x), \lambda(t), \psi(t, y)$, and $\varphi_{0}(x)$.

\section{Proposed Method: Overview of Spectral Method Formalism}

Reduce the analysis problem to the finding of Fourier coefficients $\varphi_{i_{0} i_{1} \ldots i_{n}}$ for the function $\varphi(t, x)$. Let $\left\{q\left(i_{0}, t\right)\right\}_{i_{0}=0}^{\infty}$ be an orthonormal basis of $L_{2}(T)$ and let $\left\{p\left(i_{1}, \cdots, i_{n}, x\right)\right\}_{i_{1}, \cdots, i_{n}=0}^{\infty}$ be an orthonormal basis of $L_{2}\left(R^{n}\right)$, then $\left\{e\left(i_{0}, i_{1}, \cdots, i_{n}, t, x\right)\right\}_{i_{0}, i_{1}, \cdots, i_{n}=0}^{\infty}$ is the orthonormal basis of $L_{2}\left(T \times R^{n}\right)$, where

$$
e\left(i_{0}, i_{1}, \cdots, i_{n}, t, x\right)=q\left(i_{0}, t\right) \cdot p\left(i_{1}, \cdots, i_{n}, x\right),
$$

$i_{0}, i_{1}, \cdots, i_{n}=0,1,2, \cdots$. So, 


$$
\begin{aligned}
& \varphi_{i_{0} i_{1} \cdots i_{n}}=\int_{T \times R^{n}} e\left(i_{0}, i_{1}, \cdots, i_{n}, t, x\right) \varphi(t, x) \mathrm{d} t \mathrm{~d} x, \\
& i_{0}, i_{1}, \cdots, i_{n}=0,1,2, \cdots
\end{aligned}
$$

We apply the spectral method formalism [5,8] to Equation (2) and Equation (3) subject to the conditions (5), therefore

$$
\begin{aligned}
& P(n+1, n+1) \cdot \Phi^{(1)}(n+1,0)-q\left(1,0 ; t_{0}\right) \otimes \Phi_{0}(n, 0) \\
& =A(n+1, n+1) \cdot \Phi^{(1)}(n+1,0) \\
& -\Lambda(n+1, n+1) \cdot \Phi^{(1)}(n+1,0) \\
& +H(n+1, n+1) \cdot \Phi^{(N)}(n+1,0) \\
& P(n+1, n+1) \cdot \Phi^{(k)}(n+1,0) \\
& =A(n+1, n+1) \cdot \Phi^{(k)}(n+1,0) \\
& \quad-\Lambda(n+1, n+1) \cdot \Phi^{(k)}(n+1,0) \\
& \quad+\Lambda(n+1, n+1) \cdot \Phi^{(k-1)}(n+1,0) \\
& k=2, \cdots, N
\end{aligned}
$$

In these equations $P(n+1, n+1)$ is the spectral characteristic of the differential operator $\partial / \partial t$ subject to a function value at the initial time moment $t_{0} ; A(n+1, n+1)$ and $H(n+1, n+1)$ are the spectral characteristics of operators $\mathcal{A}$ and $\mathcal{H}$ defined by (4)

and (6), respectively, i.e., $P(n+1, n+1)=\left[P_{i_{0} i_{1} \cdots i_{n} j_{0} j_{1} \cdots j_{n}}\right]$,

$$
A(n+1, n+1)=\left[A_{i_{0} i_{1} \cdots i_{n} j_{0} j_{1} \cdots j_{n}}\right]
$$

and

$$
H(n+1, n+1)=\left[H_{i_{0} i_{1} \cdots i_{n} j_{0} j_{1} \cdots j_{n}}\right]
$$

are 2(n+1)-dimensional matrices [9] (see Appendix) with elements

$$
\begin{aligned}
& P_{i_{0} i_{1} \cdots i_{n} j_{0} j_{1} \cdots j_{n}} \\
& =\int_{T \times R^{n}} e\left(i_{0}, i_{1}, \cdots, i_{n}, t, x\right) \frac{\partial e\left(j_{0}, j_{1}, \cdots, j_{n}, t, x\right)}{\partial t} \mathrm{~d} t \mathrm{~d} x \\
& \quad+q\left(i_{0}, t_{0}\right) \cdot q\left(j_{0}, t_{0}\right) \cdot\left\{\begin{array}{l}
1, i_{1}=j_{1}, \cdots, i_{n}=j_{n} \\
0, \text { otherwise }
\end{array}\right\}, \\
& A_{i_{0} i_{1} \cdots i_{n} j_{0} j_{1} \cdots j_{n}} \\
& =\int_{T \times R^{n}} e\left(i_{0}, i_{1}, \cdots, i_{n}, t, x\right) \mathcal{A} e\left(j_{0}, j_{1}, \cdots, j_{n}, t, x\right) \mathrm{d} t \mathrm{~d} x, \\
& H_{i_{0} i_{1} \cdots i_{n} j_{0} j_{1} \cdots j_{n}} \\
& =\int_{T \times R^{n}} e\left(i_{0}, i_{1}, \cdots, i_{n}, t, x\right) \mathcal{H} e\left(j_{0}, j_{1}, \cdots, j_{n}, t, x\right) \mathrm{d} t \mathrm{~d} x, \\
& i_{0}, i_{1}, \cdots, i_{n}, j_{0}, j_{1}, \cdots, j_{n}=0,1,2, \cdots ;
\end{aligned}
$$

$\Lambda(n+1, n+1)$ is the spectral characteristic of the multiplication operator with multiplier $\lambda(t)$ :

$$
\begin{aligned}
& \Lambda(n+1, n+1) \\
&= {\left[\Lambda_{i_{0} i_{1} \cdots i_{n} j_{0} j_{1} \cdots j_{n}}\right], \Lambda_{i_{0} i_{1} \cdots i_{n} j_{0} j_{1} \cdots j_{n}} } \\
&= \int_{T \times R^{n}} \lambda(t) e\left(i_{0}, i_{1}, \cdots, i_{n}, t, x\right) e\left(j_{0}, j_{1}, \cdots, j_{n}, t, x\right) \mathrm{d} t \mathrm{~d} x \\
&= \int_{T} \lambda(t) q\left(i_{0}, t\right) q\left(j_{0}, t\right) \mathrm{d} t \cdot\left\{\begin{array}{l}
1, i_{1}=j_{1}, \cdots, i_{n}=j_{n} \\
0, \text { otherwise }
\end{array}\right\}, \\
& i_{0}, i_{1}, \cdots, i_{n}, j_{0}, j_{1}, \cdots, j_{n}=0,1,2, \cdots ;
\end{aligned}
$$

$\Phi^{(k)}(n+1,0)$ are the spectral characteristics of functions $\varphi^{(k)}(t, x), k=1,2, \cdots, N$. All these spectral characteristics are defined relative to $\left\{e\left(i_{0}, i_{1}, \cdots, i_{n}, t, x\right)\right\}_{i_{0}, i_{1}, \cdots, i_{n}=0}^{\infty}$. Further, the $q\left(1,0 ; t_{0}\right)$ is the column matrix with values of functions $\left\{q\left(i_{0}, t\right)\right\}_{i_{0}=0}^{\infty}$ at the initial time moment $t_{0}$ :

$$
q\left(1,0 ; t_{0}\right)=\left[\begin{array}{lll}
q\left(0, t_{0}\right) & q\left(1, t_{0}\right) \quad q\left(2, t_{0}\right) \quad \cdots
\end{array}\right]^{\mathrm{T}} ;
$$

$\Phi_{0}(n, 0)$ is the spectral characteristic for the probability density function $\varphi_{0}(x)$ of the initial state $X_{0}$. It is defined relative to $\left\{p\left(i_{1}, \cdots, i_{n}, x\right)\right\}_{i_{1}, \cdots, i_{n}=0}^{\infty}$, i.e.,

$$
\begin{aligned}
& \Phi_{0}(n, 0)=\left[\varphi_{0 ; i_{1} \cdots i_{n}}\right], \\
& \varphi_{0 ; i_{1} \cdots i_{n}}=\int_{R^{n}} p\left(i_{1}, \cdots, i_{n}, x\right) \varphi_{0}(x) \mathrm{d} x, \\
& i_{1}, \cdots, i_{n}=0,1,2, \cdots .
\end{aligned}
$$

The spectral characteristic $\Phi(n+1,0)$ of the probability density function $\varphi(t, x)$, also called a generalized characteristic function [5,6], may be expressed as follows $(\Phi(n+1,0)$ is the $(n+1)$-multidimensional matrix formed by Fourier coefficients $\left.\varphi_{i_{0} i_{1} \cdots i_{n}}\right)$ :

$$
\begin{aligned}
& \Phi(n+1,0)=\sum_{k=1}^{N} \Phi^{(k)}(n+1,0), \\
& \Phi(n+1,0)=\left[\varphi_{i_{0} i_{1} \cdots i_{n}}\right] \\
& \Phi^{(k)}(n+1,0)=\left[\varphi_{i_{0} i_{1} \cdots i_{n}}^{(k)}\right] .
\end{aligned}
$$

The properties of the spectral characteristics for functions and linear operators in Equations (7)-(9) are described in $[5,8]$.

As a rule $[5,6]$, the spectral characteristic $A(n+1, n+1)$ is expressed in terms of the spectral characteristics for differential operators and multiplication operators:

$$
\begin{aligned}
A(n+1, n+1) \\
=-\sum_{i=1}^{n} \mathcal{P}_{i}(n+1, n+1) \cdot F_{i}(n+1, n+1) \\
\quad+\frac{1}{2} \sum_{i=1}^{n} \sum_{j=1}^{n} \mathcal{P}_{i j}(n+1, n+1) \cdot G_{i j}(n+1, n+1),
\end{aligned}
$$

where $\mathcal{P}_{i}(n+1, n+1)$ and $\mathcal{P}_{i j}(n+1, n+1)$ are the spec- 
tral characteristics of first-order and second-order differential operators $\partial / \partial x_{i}$ and $\partial^{2} / \partial x_{i} \partial x_{j}$, respectively; $F_{i}(n+1, n+1)$ and $G_{i j}(n+1, n+1)$ are the spectral characteristics of multiplication operators with multipliers $f_{i}(t, x)$ and $g_{i j}(t, x)$, respectively. These spectral characteristics are defined like a $A(n+1, n+1)$ relative to the orthonormal basis $\left\{e\left(i_{0}, i_{1}, \cdots, i_{n}, t, x\right)\right\}_{i_{0}, i_{1}, \cdots, i_{n}=0}^{\infty}$. Such definition of $A(n+1, n+1)$ is more preferred since there are analytical expressions of the spectral characteristics relative to various orthonormal functions for differential operators and multiplication operators (see $[4,5])$.

Equation (7) and Equation (8) are linear matrix equations for the spectral characteristics $\Phi^{(k)}(n+1,0)$ or linear algebraic equations for Fourier coefficients $\varphi_{i_{0} i_{1} \cdots i_{n}}^{(k)}$ (for functions $\varphi^{(k)}(t, x)$ ). Let us consider the solution of these equations.

It follows from Equation (8) that

$$
\begin{aligned}
& (P(n+1, n+1)-A(n+1, n+1)+\Lambda(n+1, n+1)) \\
& \times \Phi^{(k)}(n+1,0) \\
= & \Lambda(n+1, n+1) \cdot \Phi^{(k-1)}(n+1,0),
\end{aligned}
$$

i.e.,

$$
\begin{aligned}
& \Phi^{(k-1)}(n+1,0) \\
= & \Lambda^{-1}(n+1, n+1) \\
& \times(P(n+1, n+1)-A(n+1, n+1)+\Lambda(n+1, n+1)) \\
& \times \Phi^{(k)}(n+1,0)
\end{aligned}
$$

or

$$
\Phi^{(k-1)}(n+1,0)=W(n+1, n+1) \cdot \Phi^{(k)}(n+1,0),
$$

where

$$
\begin{aligned}
W & (n+1, n+1) \\
= & \Lambda^{-1}(n+1, n+1) \\
& \times(P(n+1, n+1)-A(n+1, n+1)+\Lambda(n+1, n+1)) \\
= & \Lambda^{-1}(n+1, n+1) \\
& \times(P(n+1, n+1)-A(n+1, n+1))+E(n+1, n+1) .
\end{aligned}
$$

Thus,

$$
\begin{aligned}
& \Phi^{(1)}(n+1,0)=W^{k-1}(n+1, n+1) \cdot \Phi^{(k)}(n+1,0), \\
& \Phi^{(k)}(n+1,0)=W^{N-k}(n+1, n+1) \cdot \Phi^{(N)}(n+1,0),
\end{aligned}
$$

in particular

$$
\Phi^{(1)}(n+1,0)=W^{N-1}(n+1, n+1) \cdot \Phi^{(N)}(n+1,0) .
$$

We rewrite Equation (7) subject to Equation (11):

$$
\begin{aligned}
( & P(n+1, n+1)-A(n+1, n+1)+\Lambda(n+1, n+1)) \\
& \times W^{N-1}(n+1, n+1) \cdot \Phi^{(N)}(n+1,0) \\
& -H(n+1, n+1) \cdot \Phi^{(N)}(n+1,0) \\
= & q\left(1,0 ; t_{0}\right) \otimes \Phi_{0}(n, 0)
\end{aligned}
$$

or

$$
\begin{aligned}
& \left(\Lambda(n+1, n+1) \cdot W^{N}(n+1, n+1)-H(n+1, n+1)\right) \\
& \times \Phi^{(N)}(n+1,0) \\
= & q\left(1,0 ; t_{0}\right) \otimes \Phi_{0}(n, 0),
\end{aligned}
$$

therefore

$$
\begin{aligned}
& \Phi^{(N)}(n+1,0) \\
= & \left(\Lambda(n+1, n+1) \cdot W^{N}(n+1, n+1)-H(n+1, n+1)\right)^{-1} \\
& \times\left(q\left(1,0 ; t_{0}\right) \otimes \Phi_{0}(n, 0)\right) .
\end{aligned}
$$

We express the spectral characteristic $\Phi(n+1,0)$ subject to Equation (9):

$$
\begin{aligned}
\Phi & (n+1,0) \\
= & \sum_{k=1}^{N} W^{N-k}(n+1, n+1) \cdot \Phi^{(N)}(n+1,0) \\
= & \left(W^{N-1}(n+1, n+1)+\cdots+W^{2}(n+1, n+1)\right. \\
& +W(n+1, n+1)+E(n+1, n+1)) \\
& \times \Phi^{(N)}(n+1,0),
\end{aligned}
$$

where $E(n+1, n+1)$ is the $2(n+1)$-dimensional identity matrix. The expression in parentheses is multiplied on the right by the difference $E(n+1, n+1)-W(n+1, n+1)$ :

$$
\begin{aligned}
&\left(W^{N-1}(n+1, n+1)+\cdots+W^{2}(n+1, n+1)\right. \\
&+W(n+1, n+1)+E(n+1, n+1)) \\
& \times(E(n+1, n+1)-W(n+1, n+1)) \\
&= W^{N-1}(n+1, n+1)+\cdots+W^{2}(n+1, n+1) \\
&+W(n+1, n+1)+E(n+1, n+1) \\
&-W^{N}(n+1, n+1)-\cdots-W^{3}(n+1, n+1) \\
&-W^{2}(n+1, n+1)-W(n+1, n+1) \\
&= E(n+1, n+1)-W^{N}(n+1, n+1),
\end{aligned}
$$

i.e.,

$$
\begin{aligned}
W^{N-1}(n+1, n+1)+\cdots+W^{2}(n+1, n+1) \\
\quad+W(n+1, n+1)+E(n+1, n+1) \\
=\left(E(n+1, n+1)-W^{N}(n+1, n+1)\right) \\
\quad \times(E(n+1, n+1)-W(n+1, n+1))^{-1} .
\end{aligned}
$$


A similar result can be obtained by multiplying on the left by $E(n+1, n+1)-W(n+1, n+1)$ :

$$
\begin{aligned}
W^{N-1}(n+1, n+1)+\cdots+W^{2}(n+1, n+1) & +W(n+1, n+1)+E(n+1, n+1) \\
= & (E(n+1, n+1)-W(n+1, n+1))^{-1} \\
& \times\left(E(n+1, n+1)-W^{N}(n+1, n+1)\right) .
\end{aligned}
$$

Equations (12) and (13), obviously, are analogues of geometric series sum. Thus,

$$
\begin{aligned}
\Phi & (n+1,0) \\
= & \left(E(n+1, n+1)-W^{N}(n+1, n+1)\right) \\
& \times(E(n+1, n+1)-W(n+1, n+1))^{-1} \\
& \times\left(\Lambda(n+1, n+1) \cdot W^{N}(n+1, n+1)\right. \\
& -H(n+1, n+1))^{-1} \times\left(q\left(1,0 ; t_{0}\right) \otimes \Phi_{0}(n, 0)\right)
\end{aligned}
$$

or

$$
\begin{aligned}
& \Phi(n+1,0) \\
&=(E(n+1, n+1)-W(n+1, n+1))^{-1} \\
& \times\left(E(n+1, n+1)-W^{N}(n+1, n+1)\right) \\
& \times\left(\Lambda(n+1, n+1) \cdot W^{N}(n+1, n+1)\right. \\
&-H(n+1, n+1))^{-1} \times\left(q\left(1,0 ; t_{0}\right) \otimes \Phi_{0}(n, 0)\right),
\end{aligned}
$$

are the problem solutions by the spectral method formalism.

It is easy to see that if $N=1$ (order of Erlang process) the problem reduces to the analysis of the stochastic systems with Poisson flow of jumps and

$$
\begin{aligned}
\Phi & (n+1,0) \\
= & (P(n+1, n+1)-A(n+1, n+1) \\
& +\Lambda(n+1, n+1)-H(n+1, n+1))^{-1} \\
& \times\left(q\left(1,0 ; t_{0}\right) \otimes \Phi_{0}(n, 0)\right) .
\end{aligned}
$$

when jump part is missed:

$$
H(n+1, n+1)=\Lambda(n+1, n+1),
$$

we obtain the known solution of analysis problem for the stochastic systems with continuous trajectories $[5,6]$ :

$$
\begin{aligned}
\Phi & (n+1,0) \\
= & (P(n+1, n+1)-A(n+1, n+1))^{-1} \\
& \times\left(q\left(1,0 ; t_{0}\right) \otimes \Phi_{0}(n, 0)\right)
\end{aligned}
$$

It is required to apply the inversion formula for finding the solution of the analysis problem [5]:

$$
\begin{aligned}
& \varphi(t, x) \\
& =\sum_{i_{0}=0}^{\infty} \sum_{i_{1}=0}^{\infty} \cdots \sum_{i_{n}=0}^{\infty} \varphi_{i_{0} i_{1} \cdots i_{n}} \cdot e\left(i_{0}, i_{1}, \cdots, i_{n}, t, x\right), \\
& (t, x) \in T \times R^{n},
\end{aligned}
$$

but a finite number of coefficients $\varphi_{i_{0} i_{1} \ldots i_{n}}$ is usually defined approximately since the problem of finding all Fourier coefficients is not trivial. In this case, the infinite matrices in Equations (7)-(9) are replaced by truncated matrices. Then

$$
\varphi(t, x) \approx \sum_{i_{0}=0}^{L_{0}-1} \sum_{i_{1}=0}^{L_{1}-1} \cdots \sum_{i_{n}=0}^{L_{n}-1} \varphi_{i_{0} i_{1} \cdots i_{n}} \cdot e\left(i_{0}, i_{1}, \cdots, i_{n}, t, x\right),
$$

where natural numbers $L_{0}, L_{1}, \cdots, L_{n}$ are the selected orders of the truncation for the spectral characteristics.

\section{Remarks}

1) The solution of the analysis problem is possible to find in another way. To do this we express $\Phi^{(k)}(n+1,0)$ in terms of $\Phi^{(k-1)}(n+1,0)$ from Equation (10), then we express $\Phi^{(k)}(n+1,0)$ in terms of $\Phi^{(1)}(n+1,0), k=2, \cdots, N$, that makes it possible to express $\Phi^{(1)}(n+1,0)$ from the Equation (7). The next step is to develop the final formula for $\Phi(n+1,0)$ subject to Equation (9) and the similar transformations carried out to express Equations (14) and (15):

$$
\begin{aligned}
\Phi & (n+1,0) \\
= & \left(Z(n+1, n+1)-Z^{N+1}(n+1, n+1)\right) \\
& \times(E(n+1, n+1)-Z(n+1, n+1))^{-1} \\
& \times\left(\Lambda(n+1, n+1)-H(n+1, n+1) \cdot Z^{N}(n+1, n+1)\right)^{-1} \\
& \times\left(q\left(1,0 ; t_{0}\right) \otimes \Phi_{0}(n, 0)\right) \\
\text { or } & \Phi(n+1,0) \\
= & (E(n+1, n+1)-Z(n+1, n+1))^{-1} \\
& \times\left(Z(n+1, n+1)-Z^{N+1}(n+1, n+1)\right) \\
& \times\left(\Lambda(n+1, n+1)-H(n+1, n+1) \cdot Z^{N}(n+1, n+1)\right)^{-1} \\
& \times\left(q\left(1,0 ; t_{0}\right) \otimes \Phi_{0}(n, 0)\right),
\end{aligned}
$$

or

where

$$
\begin{aligned}
Z & (n+1, n+1) \\
= & (P(n+1, n+1)-A(n+1, n+1)+\Lambda(n+1, n+1))^{-1} \\
& \times \Lambda(n+1, n+1) \\
= & W^{-1}(n+1, n+1) .
\end{aligned}
$$


These expressions are equivalent to Equations (14) and (15), but Equations (14) and (15) are preferable since finding the inverse spectral characteristic $\Lambda^{-1}(n+1, n+1)$ can be avoided in this case by defining $\Lambda^{-1}(n+1, n+1)$ as the spectral characteristic of the multiplication operator with multiplier $1 / \lambda(t)$. In particular, when the transition intensity is constant $(\lambda(t)=\lambda)$ we have

$$
\Lambda^{-1}(n+1, n+1)=\frac{1}{\lambda} \cdot E(n+1, n+1) .
$$

2) A generalization of the discussed problem is to consider the transition intensity which depends on the state vector. The jump size may be described by the conditional probability density function $\psi(t, x \mid z)$ that characterizes the distribution of the state vector $X\left(\tau_{i}\right)$ after the jump. This distribution depends on the previous value $X\left(\tau_{i}-0\right)=z$; jumps in sample paths of the process $X(t)$ occur at time moment $\tau_{i}$.

In this case Equations (2) and (3) are represented as

$$
\begin{aligned}
& \frac{\partial \varphi^{(1)}(t, x)}{\partial t} \\
& =\mathcal{A} \varphi^{(1)}(t, x)-\lambda(t, x) \varphi^{(1)}(t, x) \\
& +\int_{R^{n}} \lambda(t, z) \psi(t, x \mid z) \varphi^{(N)}(t, z) \mathrm{d} z, \\
& \frac{\partial \varphi^{(k)}(t, x)}{\partial t} \\
& =\mathcal{A} \varphi^{(k)}(t, x)-\lambda(t, x) \varphi^{(k)}(t, x)+\lambda(t, x) \varphi^{(k-1)}(t, x), \\
& k=2, \cdots, N,
\end{aligned}
$$

and the operator $\mathcal{H}$ (see Equation (6)) must be redefined as

$$
\mathcal{H} \varphi(t, x)=\int_{R^{n}} \lambda(t, z) \psi(t, x \mid z) \varphi(t, z) \mathrm{d} z .
$$

Equations (7) and (8) will not change (but $\Lambda(n+1, n+1)$ is the spectral characteristic of the multiplication operator with multiplier $\lambda(t, x)$, the spectral characteristic $H(n+1, n+1)$ is calculated according to a new definition of the operator $\mathcal{H}$ ). Therefore methods for the problem solution will not change as well as Equations (14) and (15).

\section{Conclusions}

We examine using of the spectral method formalism to the probabilistic analysis problem for the stochastic systems with jumps generated by Erlang flow of events. Finding of the probability density function for the state vector by the spectral method formalism are developed.

Using of Erlang flow of events allows to consider a more complex behavior for sample paths of the process $X(t)$. The occurrence of jumps in sample paths can be controlled by appropriate selection of parameters such as the transition intensity $\lambda(t)$ and an order $N$ (for Erlang process). This option makes it quite flexible tool for modeling. Thus, for $N=1$ time intervals between jumps are described by exponential distribution law, for $N>1$ time intervals between jumps are described by Erlang distribution, which is a special case of the gamma distribution. Erlang distribution converges to the normal distribution as $N$ increases.

The application of the spectral method formalism allows to reduce integro-differential equations to linear algebraic equations for Fourier coefficients of the probability density function. It essentially simplifies the solution.

\section{Acknowledgements}

This work is supported by RFBR grant 12-08-00892-a.

\section{REFERENCES}

[1] V. S. Pugachev and I. N. Sinitsyn, "Stochastic Systems: Theory and Applications," World Scientific, New Jersey, 2001.

[2] I. E. Kazakov, V. M. Artem'ev and V. A. Bukhalev, "Analysis of Systems with Random Structure," Fizmatlit, Moscow, 1993. (In Russian: И. Е. Казаков, В. М. Артемьев, В. А. Бухалев, “Анализ систем случайной структуры,” Физматлит, Москва, 1993.)

[3] F. B. Hanson, "Applied Stochastic Processes and Control for Jump-Diffusions," SIAM, Philadelphia, 2007.

[4] V. V. Solodovnikov, V. V. Semenov, M. Peschel and D. Nedo, "Design of Control Systems on Digital Computers: Spectral and Interpolational Methods," Verlag Technik, Berlin; Maschinostrojenije, Moscow, 1979. (In German: W. W. Solodownikow, W. W. Semjonow, M. Peschel and D. Nedo, "Berechnung von Regelsystemen auf Digitalrechnern: Anwendung von Spektral und Interpolationsmethoden," Verlag Technik, Berlin, 1979; In Russian: В. В. Солодовников, В. В. Семенов, М. Пешель, Д. Недо, “Расчет систем управления на ЦВМ: спектральный и интерполяционный методы," Машиностроение, Москва, 1979.)

[5] A. V. Panteleev, K. A. Rybakov and I. L. Sotskova, "Spectral Method of Nonlinear Stochastic Control System Analysis," Vuzovskaya kniga, Moscow, 2006. (In Russian: А. В. Пантелеев, К. А. Рыбаков, И. Л. Сотскова, "Спектральный метод анализа нелинейных стохастических систем управления,” Вузовская книга, Москва, 2006.)

[6] A. V. Panteleev and K. A. Rybakov, "Analyzing Nonlinear Stochastic Control Systems in the Class of Generalized Characteristic Functions," Automation and Remote Control, Vol. 72, No. 2, 2011, pp. 393-404. doi:10.1134/S0005117911020159

[7] F. A. Haight, "Handbook of the Poisson Distribution," John Wiley \& Sons, New York, 1967.

[8] K. A. Rybakov and I. L. Sotskova, "Spectral Method for Analysis of Switching Diffusions," IEEE Transactions on 
Automatic Control, Vol. 52, No. 7, 2007, pp. 1320-1325. doi:10.1109/TAC.2007.900841

[9] M. Lindner, "Infinite Matrices and their Finite Sections:
An Introduction to the Limit Operator Method," Birkhäuser, Basel, 2006.

\section{Appendix}

\section{Multidimensional Matrix Operations}

1) Let $\alpha, \beta \in R$ and let $A(p, q)=\left[a_{i_{1} \cdots i_{p} j_{1} \cdots j_{q}}\right]$ and $B(p, q)=\left[b_{i_{1} \cdots i_{p} j_{1} \cdots j_{q}}\right]$ be the infinite $(p+q)$-dimensional matrices. The expression $\alpha A(p, q)+\beta B(p, q)$ is the infinite $(p+q)$-dimensional matrix

$$
\begin{aligned}
C(p, q)= & {\left[c_{i_{1} \cdots i_{p} j_{1} \cdots j_{q}}\right] \text { if } } \\
& c_{i_{1} \cdots i_{p} j_{1} \cdots j_{q}}=\alpha a_{i_{1} \cdots i_{p} j_{1} \cdots j_{q}}+\beta b_{i_{1} \cdots i_{p} j_{1} \cdots j_{q}}, \\
& i_{1}, \cdots, i_{p}, j_{1}, \cdots, j_{q}=0,1,2, \cdots
\end{aligned}
$$

2) Let $A(p, r)=\left[a_{i_{1} \cdots i_{p} k_{1} \cdots k_{r}}\right]$ and $\left.\begin{array}{c}B(r, q) \\ =\left[b_{k_{1} \cdots k_{r} j_{1} \cdots j_{q}}\right]\end{array}\right]$ be

the infinite $(p+r)$-dimensional and $(r+q)$-dimensional matrices, respectively. The product

$A(p, r) \cdot B(r, q)$ is the infinite $(p+q)$-dimensional matrix $C(p, q)=\left[C_{i_{1} \cdots i_{p} j_{1} \cdots j_{q}}\right]$

$$
\begin{aligned}
& c_{i_{1} \cdots i_{p} j_{1} \cdots j_{q}}=\sum_{k_{1}, \cdots, k_{r}=0}^{\infty} a_{i_{1} \cdots i_{p} k_{1} \cdots k_{r}} \cdot b_{k_{1} \cdots k_{r} j_{1} \cdots j_{q}}<\infty, \\
& i_{1}, \cdots, i_{p}, j_{1}, \cdots, j_{q}=0,1,2, \cdots
\end{aligned}
$$

An infinite $2 p$-dimensional matrix $E(p, p)$ is said to be the identity matrix if

$$
A(p, p) \cdot E(p, p)=E(p, p) \cdot A(p, p)=A(p, p)
$$

for each $2 p$-dimensional matrix $A(p, p)$. We use the notation $A^{N}(p, p)$ to denote the product

$$
\begin{aligned}
& A^{N-1}(p, p) \cdot A(p, p), \\
& N=1,2, \cdots\left(A^{0}(p, p)=E(p, p)\right) .
\end{aligned}
$$

3) Let $A(p, p)$ be an infinite $2 p$-dimensional matrix. An infinite $2 p$-dimensional matrix $B(p, p)$ is said to be the two-sided inverse of $A(p, p)$ if

$$
A(p, p) \cdot B(p, p)=B(p, p) \cdot A(p, p)=E(p, p) .
$$

We use the notation $A^{-1}(p, p)$ to denote the twosided inverse of $A(p, p)$.

$$
\text { 4) Let } A(p, q)=\left[a_{i_{1} \cdots i_{p} j_{1} \cdots j_{q}}\right] \text { and } \begin{gathered}
B(r, s) \\
=\left[b_{k_{1} \cdots k_{r} l_{1} \cdots l_{s}}\right]
\end{gathered} \text { be }
$$

the infinite $(p+q)$-dimensional and $(r+s)$-dimensional matrices, respectively. The tensor product $A(p, q) \otimes B(r, s)$ is the infinite $(p+r+q+s)$-dimensional matrix $C(p+r, q+s)=\left[c_{i_{1} \cdots i_{p} k_{1} \cdots k_{r} j_{1} \cdots j_{q} l_{1} \cdots l_{s}}\right]$ if

$$
\begin{aligned}
& c_{i_{1} \cdots i_{p} k_{1} \cdots k_{r} j_{1} \cdots j_{q} l_{1} \cdots l_{s}} \\
& =a_{i_{1} \cdots i_{p} j_{1} \cdots j_{q}} \cdot b_{k_{1} \cdots k_{r} l_{1} \cdots l_{s}}, \\
& i_{1}, \cdots, i_{p}, k_{1}, \cdots, k_{r}, j_{1}, \cdots, j_{q}, l_{1}, \cdots, l_{s}=0,1,2, \cdots
\end{aligned}
$$

5) Let $A(p, q)=\left[a_{i_{1} \cdots i_{p} j_{1} \cdots j_{q}}\right]$ be an infinite $(p+q)$ -dimensional matrix. An infinite $(p+q)$-dimensional matrix $B(q, p)=\left[b_{j_{1} \cdots q_{q_{1}} \cdots i_{p}}\right]$ is said to be the transpose of $A(p, q)$ if

$$
b_{j_{1} \cdots j_{q} i_{1} \cdots i_{p}}=a_{i_{1} \cdots i_{p} j_{1} \cdots j_{q}}, i_{1}, \cdots, i_{p}, j_{1}, \cdots, j_{q}=0,1,2, \cdots
$$

We use the notation $[A(p, q)]^{\mathrm{T}}$ to denote the transpose of $A(p, q)$. 